\title{
Microwave photonic filters using optical to microwave frequency bandwidth translation with Kerr soliton crystal micro-combs
}

David Moss ( $\square$ dmoss@swin.edu.au )

Swinburne University of Technology

\section{Research Article}

Keywords: kerr microcombs, microwave, filters, photonics

Posted Date: August 26th, 2021

DOl: https://doi.org/10.21203/rs.3.rs-844249/v1

License: (c) (i) This work is licensed under a Creative Commons Attribution 4.0 International License.

Read Full License 


\section{Abstract}

We demonstrate high-resolution photonic RF filters using an RF bandwidth scaling approach based on integrated Kerr optical micro-combs. By employing both an active nonlinear micro-ring resonator (MRR) as a high-quality micro-comb source and a passive high-Q MRR to slice the shaped comb, a large RF instantaneous bandwidth of $4.64 \mathrm{GHz}$ and a high resolution of $117 \mathrm{MHz}$ are achieved, together with a broad RF operation band covering 3.28 to $19.4 \mathrm{GHz}$ ( $\mathrm{L}$ to Ku bands) using thermal tuning. We achieve programmable RF transfer functions including binary-coded notch filters and RF equalizing filters with reconfigurable slopes. Our approach is an attractive solution for high performance RF spectral shaping with high performance and flexibility.

\section{Introduction}

Radio frequency (RF) filters are one of the most basic and commonly used components in modern radar and satellite communications systems [1-5]. While electronics is subject to bandwidth bottlenecks [6], photonic techniques can realize high performance RF filters, offering not only wide bandwidths, but also low loss and strong immunity to electromagnetic interference.

Several approaches have been used for photonic RF filters, including mapping the optical filters' responses onto the RF domain, highlighted by on-chip (waveguide based) stimulated Brillouin scattering [7-9]. This approach has achieved extremely high performance in terms of RF resolution - as high as 32 $\mathrm{MHz}$ - and a stopband rejection $>55 \mathrm{~dB}$, although it faces challenges in achieving highly reconfigurable transfer functions. Another key approach is based on photonic transversal structures [10-15] that can achieve flexible and arbitrary RF transfer functions simply by changing the tap weights. However, this solution is subject to the tradeoff between resolution and operation bandwidth - for a fixed number of taps, or wavelengths, the product of the two is fixed $[6,16]$.

Alternative schemes have been demonstrated to achieve reconfigurable RF filters with both high resolution and broad operation bandwidth, including [16] using the tailored phase response of cascaded micro-ring resonators (MRRs). Although successful, it was necessary to use a large number of MRRs to achieve arbitrary transfer functions with high resolution. Recently, "RF bandwidth scaling" [17], employing a two-step approach, has been proposed. First, the RF spectra are imprinted and stretched in the optical domain, assisted by an optical frequency comb and Vernier comb filter. Then the signal is processed by a Waveshaper and compressed to the original RF bandwidth upon photo-detection. This method has enabled high-resolution RF spectral shaping, where the operation bandwidth increases linearly with the number of wavelength channels. However, the use of electro-optic combs [18-21] introduce drawbacks such as the need of high-frequency RF sources to drive the modulators. This in turn results in a limited number of wavelengths that limits the operational bandwidth.

Here, we report a high-resolution programmable photonic RF filter achieved by RF bandwidth scaling, enabled by an integrated Kerr optical micro-comb source cascaded with a passive MRR filter. Kerr micro- 
comb sources [22-29] - particularly those based on CMOS compatible platforms [30-37] - offer many advantages over traditional multi-wavelength sources, such as the potential to provide a much higher number of wavelengths with a greatly reduced footprint and complexity. Here, we experimentally demonstrate programmable arbitrary transfer functions for RF spectral shaping with an operation bandwidth of $4.64 \mathrm{GHz}$ and a resolution of $117 \mathrm{MHz}$. This was achieved by using a micro-comb source with a free spectral range (FSR) of $\sim 49 \mathrm{GHz}$ (yielding a broadband frequency comb of 80 wavelengths over the C-band and $>160$ over the C/L-bands), in combination with a passive MRR with the same FSR and $\mathrm{Q}$ factor of 1.5 million. Further, we found that the RF tuning range (controlled via thermal tuning) varies from 3.28 to $19.4 \mathrm{GHz}$ ( $\mathrm{L}$ to Ku bands). This approach is attractive for high performance photonic RF systems.

\section{li. Operation Principle}

Figure 1 shows a schematic of the high-resolution photonic RF filter. The Kerr optical frequency combs were generated in a $49 \mathrm{GHz}$ integrated MRR ( $\mathrm{Q}$ factor of 1.5 million) pumped by a continuous-wave (CW) laser. The latter was amplified by an erbium-doped fibre amplifier, with the polarization adjusted via a polarization controller to optimize the power coupled to the MRR. When the pump wavelength was swept across one of the MRR's resonances with the pump power high enough to provide sufficient parametric gain, optical parametric oscillation occurred, ultimately generating Kerr optical combs with a spacing equal to the FSR of the MRR. The generated micro-comb served as a multi-wavelength source with the power of each comb line adjusted by Waveshapers, so to achieve the designed channel weights for constructing arbitrary transfer functions.

In the wavelength range employed by the photonic RF filter, the optical frequency of the $k_{\text {th }}(k=2,3,4, \ldots)$ comb line is

$$
f_{O F C}(k)=f_{O F C}(1)+(k-1) \delta_{O F C}
$$

\section{1}

where $f_{\mathrm{OFC}}(1)$ is the frequency of the first comb line used on the red side, and $\delta_{\mathrm{OFC}}$ is the spacing between comb lines. The shaped comb lines were then passed through a phase modulator, multicasting the input RF signal onto each comb wavelength which was subsequently spectrally sampled, or sliced, by a passive MRR.

For each channel/wavelength the passive MRR introduced a power differential between the two sidebands denoted by a factor $k(0<k<1)$. The optical field of the signal after the microring resonator is then given by:

$$
E_{o p t}=E_{0}\left[J_{0}(\beta) e^{j \omega_{0} t}+J_{1}(\beta) e^{j\left(\omega_{0}-\Omega\right) t}-\sqrt{k} \cdot J_{1}(\beta) e^{j\left(\omega_{0}+\Omega\right) t}\right]
$$


where $E_{0}$ is the intensity of the optical carrier, $J_{n}(\beta)$ denote the first kind of the Bessel function with an order $n, \beta$ is the modulation depth, $w_{0}$ is the carrier's angular frequency, and $\Omega$ is the RF frequency. Then the output RF signal after the photodetector is then,

$$
I_{R F} \propto\left|E_{o p t}\right|^{2} \propto(1-\sqrt{k}) \cdot \cos \Omega t
$$

\section{3}

Thus, the power imbalance of the two optical sidebands $(k \neq 1)$ induced by the optical notch filter (i.e., the passive MRR's through-port transmission) converted the phase modulation into intensity modulation, enabling detection by the photodetector. In addition, since $k$ is a function of $w_{0}+\Omega$ and denotes the through port transmission of the high-Q resonance optical notch filter, the passive MRR therefore converts the RF phase to intensity modulation, thus achieving a narrow bandpass RF filter for high-resolution RF spectral channelizing. Alternatively it is possible to use the drop port to directly provide intensitymodulation [17]. However, this requires a separate carrier path for multi-heterodyne detection, which is less compact and less stable than our approach.

The FSR of the passive MRR was slightly different to the micro-comb MRR, and so the channelized RF spectral segments on the different comb wavelengths featured progressively staggered RF frequencies with different weights determined by the comb shaping process. The frequency of the $k_{t h}$ channelized RF spectral segment is given by:

$$
f_{R F}(k)=f_{P M R R}(k)-f_{O F C}(k)=\left[f_{P M R R}(1)-f_{O F C}(1)\right]
$$

\section{4}

where $f_{\mathrm{PMRR}}(\mathrm{k})$ is the centre frequency of the passive MRR's $k_{t h}$ notch, $\left[f_{\mathrm{PMRR}}(1)-f_{\mathrm{OFC}}(1)\right]$ is the relative spacing between the first comb line and the first filtering resonance, corresponding to the offset of the channelized RF frequencies. The quantity $\left(\delta_{\mathrm{OFC}}-\delta_{\mathrm{PMRR}}\right)$ indicates the mismatch between the comb spacing $\left(\delta_{\mathrm{OFC}}\right)$ and the FSR of the passive MRR $\left(\delta_{\mathrm{PMRR}}\right)$, which corresponds to the channelized RF frequency step between adjacent wavelength channels.

Finally, the weighted RF spectra segments were combined upon photo-detection, thus achieving RF bandwidth scaling for arbitrary RF spectral shaping by employing the designed channel weights. Here, the resolution was determined by the passive MRR's $\mathrm{Q}$ factor and the operation bandwidth by the product of the resolution and the number of wavelengths.

\section{lii. Experimental Results}

The integrated MRRs were fabricated on a high-index doped silica glass $(\mathrm{n}=\sim 1.7$ at $1550 \mathrm{~nm})$ platform using CMOS-compatible fabrication processes, featuring ultra-low linear loss $\left(\sim 0.06 \mathrm{~dB} \bowtie \mathrm{cm}^{-1}\right)$, a moderate nonlinear parameter $\left(\sim 233 \mathrm{~W}^{-1} \triangle \mathrm{km}^{-1}\right)$, and, in particular, negligible nonlinear loss up to 
extremely high intensities ( $\left.25 \mathrm{GW} \otimes \mathrm{cm}^{-2}\right)$. Due to the ultra-low loss of our platform, the MRRs feature narrow resonance linewidths, corresponding to a Q factor of 1.5 million. After packaging the MRRs with fiber pigtails, the through-port insertion loss was $<2 \mathrm{~dB}$, assisted by on-chip mode converters. The radii of the MRRs were both designed to be $\sim 592 \mu \mathrm{m}$, corresponding to FSRs of $\sim 0.4 \mathrm{~nm}(\sim 49 \mathrm{GHz})$. To realise a mismatch between the comb spacing $\left(\delta_{\mathrm{OFC}}\right)$ and the FSR of the passive MRR $\left(\delta_{\mathrm{PMRR}}\right)$ for RF spectral channelizing, we used orthogonally polarization modes $[38,39]$ of the active and passive MRRs (TE mode of the active MRR, TM mode of the passive MRR) since they had slightly different effective indices - a result of the non-symmetric waveguide cross sections $(\sim 3 \mu \mathrm{m} \times 2 \mu \mathrm{m})$ that lead to polarization dependent FSRs.

To generate Kerr micro-combs, the pump power was set at $\sim 30.5 \mathrm{dBm}$ and the wavelength was swept across a TE resonance of the active MRR at $\sim 1552.9 \mathrm{~nm}$. When the detuning between the pump wavelength and the MRR's cold resonance became small enough, such that the intra-cavity power reached a threshold value, modulation instability driven oscillation was initiated [27]. As the detuning was changed further, distinctive 'fingerprint' optical spectra were observed (Fig. 2(a)). These spectra are similar to what has been reported from spectral interference between tightly packed solitons in the cavity - so called "soliton crystals" [28, 29, 40]. An abrupt step in the measured intracavity power (Fig. 2(b)) was observed at the point where these spectra appeared, as well as a dramatic reduction in the RF intensity noise (Fig. 2(c)). We note that it was not necessary to specifically achieve a single soliton state in order to obtain high performance-only that the chaotic regime [27] should be avoided. This is important since there is a much wider range of coherent low noise states that are more readily accessible than single soliton states [27].

The soliton crystal comb was then spectrally shaped by two stages of Waveshapers (Finisar, 4000S) in order to enable a larger dynamic range of loss control and higher shaping accuracy than a single stage $[41,42]$. The micro-comb was first pre-shaped to reduce the power difference between the comb lines to less than $15 \mathrm{~dB}$, then amplified by an erbium-doped fibre amplifier and accurately shaped by a subsequent Waveshaper according to the designed channel weights. For each Waveshaper, a feedback control path was adopted to increase the accuracy of the comb shaping, where the power in the comb lines was detected by an optical spectrum analyzer and compared with the ideal tap weights in order to generate error signals for calibration.

Then the input RF signal was multi-cast onto each comb line via a phase modulator and fed to the passive MRR for spectral slicing. The TM mode through-port transmission of the passive MRR was employed to perform phase-to-intensity modulation conversion by filtering out the lower sideband and, at the same time, map its high-Q resonances (notches) onto the RF domain for high-resolution RF filtering. The optical transmission spectrum of the passive MRR showed an FSR of $\sim 49 \mathrm{GHz}(\sim 0.4 \mathrm{~nm}$, Fig. 3(a)) and 3-dB bandwidth of $\sim 0.84 \mathrm{pm}(\sim 105 \mathrm{MHz}$, Fig. 3(b)). We note here that the measured extinction ratio as shown in Fig. 3 was limited by the resolution of our measuring system $(\sim 0.1 \mathrm{pm}$, or $10 \mathrm{MHz})$. The actual extinction ratio was greater than $20 \mathrm{~dB}$, as confirmed by the RF transmission results, measured by a vector network analyser with a resolution of $1 \mathrm{kHz}$. 
To illustrate the principle of operation of our high-resolution RF filter, we plot the flattened microcomb spectrum (TE mode) and the drop-port transmission spectrum of the passive MRR (TM mode, measured by using the ASE spectrum of an optical amplifier) in Fig. 4(a). The zoom-in views show the relative shift between the comb lines and the resonances of the passive MRR across the $C$ band, - i.e., the spectrally sliced RF frequencies $f_{\mathrm{RF}}(\mathrm{k})$ - ranging from 2.8 to $8.0 \mathrm{GHz}$. A linear fit of the extracted peak frequencies fopt (Fig. 4(b)) determines the FSRs measured to be 48.965 for the passive MRR (TM mode) and 48.898 $\mathrm{GHz}$ for the microcomb (TE mode), respectively. Further, it finds a channelized RF frequency step between adjacent channels $\left(\delta_{\mathrm{OFC}}-\delta_{\mathrm{PMRR}}\right)$ of $\sim 66.6 \mathrm{MHz}$ per channel.

To independently verify this, we measured the RF transmission (Fig. 5) of our filter at the extreme optical bandwidth limits (1st and 80th comb lines, Fig. 5, inset) using a swept frequency RF source with an RF spectrum analyser. Note that the RF bandwidth of each peak is given by the resolution of our system. The spurious lines were a result of the RF source's relatively large frequency step and did not reflect the device performance. The $4.64 \mathrm{GHz}$ spacing between the two passbands indicates an RF frequency step of 4.64 $\mathrm{GHz} / 80=58 \mathrm{MHz}$, in reasonable agreement with the optical measurements.

Finally, the optical signal was converted back into the RF domain by a high-speed photodetector, where the channelized RF spectral segments were combined upon photo-detection. To evaluate the enhanced operation bandwidth enabled by the micro-comb's large number of wavelength channels, we measured the RF transmission spectra with different number of wavelength channels (with flattened comb lines). The single channel case (Fig. 6, blue line) shows a 3dB bandwidth of $117 \pm 25 \mathrm{MHz}$ (the $\pm 25 \mathrm{MHz}$ error bar arises from the resolution of the Vector Network Analyser), which closely matches the measured linewidth of the passive MRR's resonance and defines the resolution of our RF filter. This approach requires dramatically fewer channels, or wavelengths, to achieve this high resolution, unlike transversal filters $[43,44]$ that require over 800 wavelengths (taps) to reach a similar resolution (with a Nyquist frequency of $20 \mathrm{GHz})$. Our RF frequency step $(58 \mathrm{MHz})$ was about half of the resolution $\left(\delta_{\mathrm{OFC}}-\delta_{\mathrm{PMRR}}=\right.$ $117 \mathrm{MHz}$ ), whereas ideally, they should be equal. In principle this can be achieved by lithographic control of the waveguide dimensions to vary the polarization dependent FSRs.

From Fig. 6 we see that, as the channel number increased from 20 to 80 , the operation bandwidth broadened by a factor of four from $1.17 \mathrm{GHz}$ (Fig. 6, yellow line) to $4.64 \mathrm{GHz}$ (Fig. 6, purple line). In addition, employing $\mathrm{C}+\mathrm{L}$ band Waveshapers and optical amplifiers would yield 160 wavelength channels, leading to a wide instantaneous bandwidth $>15 \mathrm{GHz}$ with a similarly high resolution of 117 $\mathrm{MHz}$. The smaller roll-off rate at the high RF frequency side of the wide bandwidth filter results from spurious phase-to-intensity modulation conversion induced by the passive MRR's phase jump, as well as any gain variation with wavelength of the optical amplifier. To minimize these effects, the passive MRR coupling can be optimized to minimize this phase jump, and the gain profile of the optical amplifier equalized with a gain flattening filter.

To demonstrate arbitrary RF transfer functions, we varied the channel weights and measured the RF transmission spectra with a vector network analyser. Figure 7 shows a reconfigurable notch filter 
achieved with binary (i.e., either " 1 " or " 0 ") channel weights. The RF transmission spectra (Fig. 7, blue line) match very well with the shaped comb spectra (Fig. 7, red line). Figure 8 shows distinctive RF transmission spectra of RF equalizing filters with flat, quadratic, positive, and negative slopes, implemented by shaping the comb spectra. The RF transmission spectra agree well with the shaped comb spectra, proving the reconfigurability and resolution of our photonic RF filter. We note that flat-top passive optical filters using cascaded MRRs [45-47] can be employed to reduce the passband ripple. The wavelength dependence of the passive MRR loss, RF modulator response, optical amplifiers, and photodetector across the $\mathrm{C}$-band created errors in the channel weights and hence did affect the RF response of the device. This can be compensated for by re-adjusting the channel weights in the Waveshaper by, for example, using an algorithm-driven feedback control path with error signals calculated directly from the generated RF transmission spectra (versus the optical spectra of the shaped comb).

To achieve broadband RF tunability, we thermally tuned the passive MRR to continuously change the relative spacing between the comb lines and passive MRR resonances $\left(f_{\mathrm{PMRR}}(1)-f_{\mathrm{OFC}}(1)\right)$, where millisecond thermal response times are achievable [48]. Figure 9 shows the measured RF transmission spectra when the chip temperature was varied from $36^{\circ} \mathrm{C}$ to $44^{\circ} \mathrm{C}$, resulting in a wideband RF tunability of 3.3 to $19.4 \mathrm{GHz}$. By employing a thermal tuning range equivalent to the FSR of the microcomb $(\sim 49 \mathrm{GHz})$ and using multiple-FSR spaced filter resonances, an ultra-wideband RF operation of $>100 \mathrm{GHz}$ can be achieved.

This work highlights the potential for microcombs in microwave signal processing [49-87], particularly in the Hydex glass platform [88-99], including recent results in optical communications [101] and neural networks [102, 103] as well as quantum optics [104-112]. Integrating devices such as detectors [113] and dispersion compensation delays on-chip [114-117] will further enhance the power and greatly reduce the footprint of these systems.

\section{Iv. Conclusion}

We demonstrate a high-resolution photonic RF filter based on RF bandwidth scaling by cascading a Kerr optical micro-combs source with a passive high-Q MRR for spectral slicing. A large RF instantaneous bandwidth of $4.64 \mathrm{GHz}$ is achieved using $80 \mathrm{comb}$ lines over the C-band, with a high resolution of 117 $\mathrm{MHz}$ and wide RF tuning range from 3-19 GHz (L-Ku bands) via thermal tuning. We achieve programmable RF transfer functions including bandpass and RF equalizing filters with reconfigurable slopes. This represents an attractive approach towards realizing high performance RF filters for modern photonic RF systems.

\section{Declarations}

\section{Competing interests:}


The authors declare no competing interests.

\section{References}

1. J. Capmany, and D. Novak, Nat. Photonics 1, 319 (2007).

2. J. P. Yao, J. Lightwave Technol. 27, 314 (2009).

3. R. C. Williamson, and R. D. Esman, J. Lightwave Technol. 26, 1145 (2008).

4. V. R. Supradeepa, C. M. Long, R. Wu, F. Ferdous, E. Hamidi, D. E. Leaird, and A. M. Weiner, Nat. Photonics 6, 186 (2012).

5. R. A. Minasian, IEEE J. Quantum Electron. 52, 0600813 (2016).

6. J. Capmany, B. Ortega, and D. Pastor, J. Lightwave Technol. 24, 201 (2006).

7. Y. Liu, Y. Yu, S. X. Yuan, X. B. Xu, and X. L. Zhang, Opt. Lett. 41, 5078 (2016).

8. D. Marpaung, B. Morrison, M. Pagani, R. Pant, D. Y. Choi, B. Luther-Davies, S. J. Madden, and B. J. Eggleton, Optica 2, 76 (2015).

9. A. Choudhary, B. Morrison, I. Aryanfar, S. Shahnia, M. Pagani, Y. Liu, K. Vu, S. Madden, D. Marpaung, and B. J. Eggleton, J. Lightwave Technol. 35, 846 (2017).

10. X. Xu, J. Wu, M. Shoeiby, T. G. Nguyen, S. T. Chu, B. E. Little, R. Morandotti, A. Mitchell, and D. J. Moss, APL Photonics 2096104 (2017).

11. X. Q. Zhu, F. Y. Chen, H. F. Peng, and Z. Y. Chen, Opt. Express 25, 9232 (2017).

12. F. Jiang, Y. Yu, H. T. Tang, L. Xu, and X. L. Zhang, Opt. Express 24 18655 (2016).

13. J. Wu, X. Xu, T. G. Nguyen, S. T. Chu, B. E. Little, R. Morandotti, A. Mitchell, and D. J. Moss, IEEE J. Sel. Top. Quantum Electron. 24, 6101020 (2018).

14. E. Hamidi, D. E. Leaird, and A. M. Weiner, IEEE Journal of Microwave Theory $\underline{\mathbf{5 8}}, 3269$ (2010).

15. Z. J. Zhu, H. Chi, T. Jin, S. L. Zheng, X. F. Jin, and X. M. Zhang, Opt. Lett. 42, 3012 (2017).

16. H. Y. Jiang, L. S. Yan, and D. Marpaung, Opt. Lett. 43, 415 (2018).

17. J. L. Li, Y. T. Dai, F. F. Yin, W. Li, M. Li, H. W. Chen, and K. Xu, Opt. Lett. 43, 1878 (2018).

18. R. Wu, V. R. Supradeepa, C. M. Long, D. E. Leaird, and A. M. Weiner, Opt. Lett. 35, 3234 (2010).

19. W. Z. Li, and J. P. Yao, Opt. Express 17, 23712 (2009).

20. C. H. Chen, C. He, D. Zhu, R. H. Guo, F. Z. Zhang, and S. L. Pan, Opt. Lett. 38, 3137 (2013).

21. T. Saitoh, M. Kourogi, and M. Ohtsu, IEEE Photonic Tech. L. 8, 1543 (1996).

22. P. Del'Haye, A. Schliesser, O. Arcizet, T. Wilken, R. Holzwarth, and T. J. Kippenberg, Nature 450, 1214 (2007).

23. T. J. Kippenberg, R. Holzwarth, and S. A. Diddams, Science 332, 555 (2011).

24. L. Razzari, D. Duchesne, M. Ferrera, R. Morandotti, S. Chu, B. E. Little, and D. J. Moss, Nat. Photonics 4, 41 (2010).

25. D. J. Moss, R. Morandotti, A. L. Gaeta, and M. Lipson, Nat. Photonics Z 597 (2013). 
26. J. S. Levy, A. Gondarenko, M. A. Foster, A. C. Turner-Foster, A. L. Gaeta, and M. Lipson, Nat. Photonics 4, 37 (2010).

27. A. Pasquazi, M. Peccianti, L. Razzari, D. J. Moss, S. Coen, M. Erkintalo, Y. K. Chembo, T. Hansson, S. Wabnitz, P. Del'Haye, X. X. Xue, A. M. Weiner, and R. Morandotti, Physics Reports $\underline{729}, 1$ (2018).

28. D. C. Cole, E. S. Lamb, P. Del'Haye, S. A. Diddams, and S. B. Papp, Nat. Photonics 11, 671 (2017).

29. W. Q. Wang, Z. Z. Lu, W. F. Zhang, S. T. Chu, B. E. Little, L. R. Wang, X. P. Xie, M. L. Liu, Q. H. Yang, L. Wang, J. G. Zhao, G. X. Wang, Q. B. Sun, Y. S. Liu, Y. S. Wang, and W. Zhao, Opt. Lett. 43, 2002 (2018).

30. M. Ferrera, C. Reimer, A. Pasquazi, M. Peccianti, M. Clerici, L. Caspani, S. T. Chu, B. E. Little, R. Morandotti, and D. J. Moss, Opt. Express 22, 21488 (2014).

31. B. Corcoran, T. D. Vo, M. D. Pelusi, C. Monat, D. X. Xu, A. Densmore, R. B. Ma, S. Janz, D. J. Moss, and B. J. Eggleton, Opt. Express 18, 20190 (2010).

32. X. Xu, J. Wu, T. G. Nguyen, T. Moein, S. T. Chu, B. E. Little, R. Morandotti, A. Mitchell, and D. J. Moss, Photonics Research $\underline{6}$, B30 (2018).

33. A. Pasquazi, L. Caspani, M. Peccianti, M. Clerici, M. Ferrera, L. Razzari, D. Duchesne, B. E. Little, S. T. Chu, D. J. Moss, and R. Morandotti, Opt. Express 21 13333 (2013).

34. A. Pasquazi, R. Ahmad, M. Rochette, M. Lamont, B. E. Little, S. T. Chu, R. Morandotti, and D. J. Moss, Opt. Express 18, 3858 (2010).

35. M. Peccianti, M. Ferrera, L. Razzari, R. Morandotti, B. E. Little, S. T. Chu, and D. J. Moss, Opt. Express 18, 7625 (2010).

36. X. Xu, J. Wu, T. G. Nguyen, S. T. Chu, B. E. Little, R. Morandotti, A. Mitchell, and D. J. Moss, J. Lightwave Technol. 36, 4519 (2018).

37. A. Pasquazi, M. Peccianti, B. E. Little, S. T. Chu, D. J. Moss, and R. Morandotti, Opt. Express 20, 27355 (2012).

38. X. Xu, J. Wu, M. X. Tan, T. G. Nguyen, S. T. Chu, B. E. Little, R. Morandotti, A. Mitchell, and D. J. Moss, J. Lightwave Technol. $\underline{\text { 36, }} 4808$ (2018).

39. X. Xu, J. Wu, L. N. Jia, M. X. Tan, T. G. Nguyen, M. Shoeiby, S. T. Chu, B. E. Little, R. Morandotti, A. Mitchell, and D. J. Moss, Journal of Optics 20, 115710 (2018).

40. X. Xu, M. Tan, J. Wu, T.G. Nguyen, S.T. Chu, B.E. Little, R. Morandotti, A. Mitchell, and D. J. Moss, "Microcomb based photonic local oscillator for broadband microwave frequency conversion", Paper Th3C.2, IEEE/OSA Optical Fiber Communications Conference, San Diego CA., March (2019) DOI:10.1364/OFC.2019.Th3C.2.

41. X. X. Xue, Y. Xuan, H. J. Kim, J. Wang, D. E. Leaird, M. H. Qi, and A. M. Weiner, J. Lightwave Technol. 32, 3557 (2014).

42. X. Xu, M. X. Tan, J. Wu, T. G. Nguyen, S. T. Chu, B. E. Little, R. Morandotti, A. Mitchell, and D. J. Moss, "Advanced adaptive photonic RF filters with 80 taps based on an integrated optical micro-comb source," J. Lightwave Technol. 37 (4) 1288-1295 (2019). DOI:10.1109/JLT.2019.2892158. 
43. X. Xu, J. Wu, T. G. Nguyen, M. Shoeiby, S. T. Chu, B. E. Little, R. Morandotti, A. Mitchell, and D. J. Moss, Opt. Express 26, 2569 (2018).

44. T. G. Nguyen, M. Shoeiby, S. T. Chu, B. E. Little, R. Morandotti, A. Mitchell, and D. J. Moss, Opt. Express $\underline{23}, 22087$ (2015).

45. J. Wu, T. Moein, X. Xu, G. H. Ren, A. Mitchell, and D. J. Moss, APL Photonics 2 , 056103 (2017).

46. B. E. Little, S. T. Chu, H. A. Haus, J. Foresi, and J.-P. Laine, J. Lightwave Technol. 15 998 (1997).

47. J. Wu, T. Moein, X. Xu, and D. J. Moss, APL Photonics $\underline{3}, 046102$ (2018).

48. X. X. Xue, Y. Xuan, C. Wang, P. H. Wang, Y. Liu, B. Niu, D. E. Leaird, M. H. Qi, and A. M. Weiner, Opt. Express 24, 687 (2016).

49. Mengxi Tan, X. Xu, J. Wu, T. G. Nguyen, S. T. Chu, B. E. Little, R. Morandotti, A. Mitchell, and David J. Moss, "Photonic Radio Frequency Channelizers based on Kerr Optical Micro-combs", Journal of Semiconductors42 (4), 041302 (2021). (ISSN 1674-4926). DOI:10.1088/1674-4926/42/4/041302.

50. H.Bao, L.Olivieri, M.Rowley, S.T. Chu, B.E. Little, R.Morandotti, D.J. Moss, J.S.T. Gongora, M.Peccianti and A.Pasquazi, "Laser Cavity Solitons and Turing Patterns in Microresonator Filtered Lasers: properties and perspectives", Paper No. LA203-5, Paper No. 11672-5, SPIE LASE, SPIE Photonics West, San Francisco CA March 6-11 (2021). DOI:10.1117/12.2576645

51. Mengxi Tan, X. Xu, J. Wu, A. Boes, T. G. Nguyen, S. T. Chu, B. E. Little, R. Morandotti, A. Mitchell, and David J. Moss, "Advanced microwave signal generation and processing with soliton crystal microcombs", or "Photonic convolutional accelerator and neural network in the Tera-OPs regime based on Kerr microcombs", Paper No. 11689-38, PW210-OE201-67, Integrated Optics: Devices, Materials, and Technologies XXV, SPIE Photonics West, San Francisco CA March 6-11 (2021). DOI: $10.1117 / 12.2584017$

52. Mengxi Tan, Bill Corcoran, Xingyuan Xu, Andrew Boes, Jiayang Wu, Thach Nguyen, Sai T. Chu, Brent E. Little, Roberto Morandotti, Arnan Mitchell, and David J. Moss, "Optical data transmission at 40 Terabits/s with a Kerr soliton crystal microcomb", Paper No.11713-8, PW210-0E803-23, NextGeneration Optical Communication: Components, Sub-Systems, and Systems X, SPIE Photonics West, San Francisco CA March 6-11 (2021). DOI:10.1117/12.2584014

53. Mengxi Tan, X. Xu, J. Wu, A. Boes, T. G. Nguyen, S. T. Chu, B. E. Little, R. Morandotti, A. Mitchell, and David J. Moss, "RF and microwave photonic, fractional differentiation, integration, and Hilbert transforms based on Kerr micro-combs", Paper No. 11713-16, PW210-OE803-24, Next-Generation Optical Communication: Components, Sub-Systems, and Systems X, SPIE Photonics West, San Francisco CA March 6-11 (2021). DOI:10.1117/12.2584018

54. Mengxi Tan, X. Xu, J. Wu, A. Boes, T. G. Nguyen, S. T. Chu, B. E. Little, R. Morandotti, A. Mitchell, and David J. Moss, "Broadband photonic RF channelizer with 90 channels based on a soliton crystal microcomb", or "Photonic microwave and RF channelizers based on Kerr micro-combs", Paper No. 11685-22, PW210-OE106-49, Terahertz, RF, Millimeter, and Submillimeter-Wave Technology and Applications XIV, SPIE Photonics West, San Francisco CA March 6-11 (2021).

DOI:10.1117/12.2584015

Page $10 / 20$ 
55. X. Xu, M. Tan, J. Wu, S. T. Chu, B. E. Little, R. Morandotti, A. Mitchell, B. Corcoran, D. Hicks, and D. J. Moss, "Photonic perceptron based on a Kerr microcomb for scalable high speed optical neural networks", IEEE Topical Meeting on Microwave Photonics (MPW), pp. 220-224,.Matsue, Japan, November 24-26, 2020. Electronic ISBN:978-4-88552-331-1.

DOI: 10.23919/MWP48676.2020.9314409

56. Mengxi Tan, Bill Corcoran, Xingyuan Xu, Andrew Boes, Jiayang Wu, Thach Nguyen, S.T. Chu, B. E. Little, Roberto Morandotti, Arnan Mitchell, and David J. Moss, "Ultra-high bandwidth optical data transmission with a microcomb”, IEEE Topical Meeting on Microwave Photonics (MPW), pp. 7882.Virtual Conf., Matsue, Japan, November 24-26, 2020. Electronic ISBN:978-4-88552-331-

1. DOI: 10.23919/MWP48676.2020.9314476

57. M. Tan, X. Xu, J. Wu, R. Morandotti, A. Mitchell, and D. J. Moss, "RF and microwave high bandwidth signal processing based on Kerr Micro-combs", Advances in Physics X, VOL. 6, NO. 1, 1838946 (2020). DOI:10.1080/23746149.2020.1838946.

58. Mengxi Tan, Xingyuan Xu, Jiayang Wu, Thach G. Nguyen, Sai T. Chu, Brent E. Little, Roberto Morandotti, Arnan Mitchell, and David J. Moss, "Photonic Radio Frequency Channelizers based on Kerr Micro-combs and Integrated Micro-ring Resonators", JOSarXiv.202010.0002.

59. Mengxi Tan, Xingyuan Xu, David Moss “Tunable Broadband RF Photonic Fractional Hilbert Transformer Based on a Soliton Crystal Microcomb”, Preprints, DOI: 10.20944/preprints202104.0162.v1

60. Mengxi Tan, X. Xu, J. Wu, T. G. Nguyen, S. T. Chu, B. E. Little, R. Morandotti, A. Mitchell, and David J. Moss, "Orthogonally polarized Photonic Radio Frequency single sideband generation with integrated micro-ring resonators", Journal of Semiconductors42 (4), 041305 (2021). DOI: 10.1088/16744926/42/4/041305.

61. H. Bao, et al., "Laser cavity-soliton microcombs," Nature Photonics, vol. 13, no. 6, pp. 384-389, Jun. 2019.

62. X. Xue, X. Zheng, and B. Zhou, "Super-efficient temporal solitons in mutually coupled optical cavities," Nature Photonics, May 2019.

63. H. Bao et al., "Turing patterns in a fibre laser with a nested micro-resonator: robust and controllable micro-comb generation”, Physical Review Research, vol. 2, pp. 023395, 2020.

64. L. D. Lauro, J. Li, D. J. Moss, R. Morandotti, S. T. Chu, M. Peccianti, and A. Pasquazi, "Parametric control of thermal self-pulsation in micro-cavities," Opt. Lett. vol. 42, no. 17, pp. 3407-3410, Aug. 2017.

65. H. Bao, et al., "Type-Il micro-comb generation in a filter-driven four wave mixing laser," Photonics Research, vol. 6, no. 5, pp. B67-B73, 2018.

66. T. G. Nguyen et al., "Integrated frequency comb source-based Hilbert transformer for wideband microwave photonic phase analysis," Opt. Express, vol. 23, no. 17, pp. 22087-22097, Aug. 2015.

67. X. Xue, et al., "Programmable single-bandpass photonic RF filter based on a Kerr comb from a microring," Journal of Lightwave Technol., vol. 32, no. 20, pp. 3557-3565, Oct. 2014. 
68. X. Xu et al., "Reconfigurable broadband microwave photonic intensity differentiator based on an integrated optical frequency comb source," APL Photonics, vol. 2, no. 9, 096104, Sep. 2017.

69. X. Xu, M. Tan, J. Wu, R. Morandotti, A. Mitchell, and D. J. Moss, "Microcomb-based photonic RF signal processing", IEEE Photonics Technology Letters, vol. 31 no. 23 1854-1857, 2019.

70. X. Xu, et al., "Advanced RF and microwave functions based on an integrated optical frequency comb source," Opt. Express, vol. 26, no. 3, pp. 2569-2583, Feb. 2018.

71. X. Xue, et al., "Microcomb-based true-time-delay network for microwave beamforming with arbitrary beam pattern control," Journal of Lightwave Technology, vol. 36, no. 12, pp. 2312-2321, Jun. 2018.

72. X. Xu, et al., "Broadband RF channelizer based on an integrated optical frequency Kerr comb source," Journal of Lightwave Technology, vol. 36, no. 19, pp. 4519-4526, 2018.

73. X. Xu, et al., "Continuously tunable orthogonally polarized RF optical single sideband generator based on micro-ring resonators," Journal of Optics, vol. 20, no. 11, pp. 115701. 2018.

74. X. Xu, et al., "Orthogonally polarized RF optical single sideband generation and dual-channel equalization based on an integrated microring resonator," Journal of Lightwave Technology, vol. 36, no. 20, pp. 4808-4818. 2018.

75. X. Xu, et al., "Photonic microwave true time delays for phased array antennas using a $49 \mathrm{GHz}$ FSR integrated optical micro-comb source," Photonics Res, vol. 6, no. 5, pp. B30-B36, 2018.

76. X. Xu, et al., "Advanced adaptive photonic RF filters with 80 taps based on an integrated optical micro-comb source," Journal of Lightwave Technology, vol. 37, no. 4, pp. 1288-1295, 2019.

77. W. Liang, et al., "High spectral purity Kerr frequency comb radio frequency photonic oscillator," Nature Comm., vol. 6 pp. 7957. 2015.

78. J. Liu, et al., "Photonic microwave generation in the X-and K-band using integrated soliton microcombs" Nature Phot., vol. 14, pp. 1-6, 2020.

79. X. Xu, et al., Broadband microwave frequency conversion based on an integrated optical micro-comb source", Journal of Lightwave Technology, vol. 38 no. 2, pp. 332-338, 2020.

80. M. Tan, et al., "Photonic RF and microwave filters based on $49 \mathrm{GHz}$ and $200 \mathrm{GHz}$ Kerr microcombs", Optics Comm. vol. 465, 125563. 2020.

81. X. Xu, et al., "Broadband photonic RF channelizer with 90 channels based on a soliton crystal microcomb", Journal of Lightwave Technology, vol. 38, no. 18, 5116 - 5121, Sept. 15 (2020). doi: 10.1109/JLT.2020.2997699

82. X. Xu, et al., "Photonic RF and microwave integrator with soliton crystal microcombs", IEEE Transactions on Circuits and Systems II: Express Briefs, vol. 67, no. 12, pp. 3582-3586, 2020.

83. X.Xu, et al.,"Photonic RF phase-encoded signal generation with a microcomb source”, J.Lightwave Technology, vol.38 (7) pp.1722-1727, 2020.

84. X. Xu, et al., "High performance RF filters via bandwidth scaling with Kerr micro-combs," APL Photonics, vol. 4, no. 2, pp. 026102. 2019. 
85. M. Tan, et al., "Microwave and RF photonic fractional Hilbert transformer based on a $50 \mathrm{GHz}$ Kerr micro-comb", Journal of Lightwave Technology, vol. 37, no. 24, pp. 6097 - 6104, 2019.

86. M. Tan, et al., "RF and microwave fractional differentiator based on photonics", IEEE Transactions on Circuits and Systems: Express Briefs, vol. 67, no.11, pp. 2767-2771, 2020.

87. M. Tan, et al., "Photonic RF arbitrary waveform generator based on a soliton crystal micro-comb source", Journal of Lightwave Technology, vol. 38, no. 22, pp. 6221-6226, Oct 22, 2020.

88. M. Ferrera, L. Razzari, D. Duchesne, et al., "Low-power continuous-wave nonlinear optics in doped silica glass integrated waveguide structures," Nature Photonics, vol. 2, no. 12, pp. 737-740, 2008.

89. A. Pasquazi, M. Peccianti, Y. Park, B. E. Little, S. T. Chu, R. Morandotti, J. Azaña, and D. J. Moss, “Subpicosecond phase-sensitive optical pulse characterization on a chip", Nature Photonics, vol. 5, no. 10, pp. $618-623(2011)$.

90. D. Duchesne, M. Peccianti, M. R. E. Lamont, et al., "Supercontinuum generation in a high index doped silica glass spiral waveguide," Optics Express, vol. 18, no, 2, pp. 923-930, 2010.

91. M. Ferrera, et al., "On-chip CMOS-compatible all-optical integrator”, Nature Communications, vol. 1, Article 29, 2010.

92. A. Pasquazi, et al., "All-optical wavelength conversion in an integrated ring resonator," Optics Express, vol. 18, no. 4, pp. 3858-3863, 2010.

93. A. Pasquazi, Y. Park, J. Azana, et al., "Efficient wavelength conversion and net parametric gain via Four Wave Mixing in a high index doped silica waveguide," Optics Express, vol. 18, no. 8, pp. 7634$7641,2010$.

94. M. Peccianti, M. Ferrera, L. Razzari, et al., "Subpicosecond optical pulse compression via an integrated nonlinear chirper," Optics Express, vol. 18, no. 8, pp. 7625-7633, 2010.

95. D. Duchesne, M. Ferrera, L. Razzari, et al., "Efficient self-phase modulation in low loss, high index doped silica glass integrated waveguides," Optics Express, vol. 17, no. 3, pp. 1865-1870, 2009.

96. A. Pasquazi, M. Peccianti, L. Razzari, D. J. Moss, S. Coen, M. Erkintalo, Y. K. Chembo, T. Hansson, S. Wabnitz, P. Del'Haye, X. X. Xue, A. M. Weiner, and R. Morandotti, “Micro-combs: A novel generation of optical sources," Physics Reports, vol. 729, pp. 1-81, Jan 27. 2018.

97. M. Peccianti, et al., "Demonstration of an ultrafast nonlinear microcavity modelocked laser", Nature Communications, vol. 3, pp. 765, 2012.

98. M. Kues, et al., "Passively modelocked laser with an ultra-narrow spectral width", Nature Photonics, vol. 11, no. 3, pp. 159, 2017.

99. A. Pasquazi, L. Caspani, M. Peccianti, et al., "Self-locked optical parametric oscillation in a CMOS compatible microring resonator: a route to robust optical frequency comb generation on a chip," Optics Express, vol. 21, no. 11, pp. 13333-13341, 2013.

100. A. Pasquazi, M. Peccianti, B. E. Little, et al., "Stable, dual mode, high repetition rate mode-locked laser based on a microring resonator," Optics Express, vol. 20, no. 24, pp. 27355-27362, 2012. 
101. B. Corcoran, et al., "Ultra-dense optical data transmission over standard fiber with a single chip source", Nature Communications, vol. 11, Article:2568, 2020.

102. X. Xu, et al., "Photonic perceptron based on a Kerr microcomb for scalable high speed optical neural networks", Laser and Photonics Reviews, vol. 14, no. 8, 2020. DOl:10.1002/Ipor.202000070.

103. X. Xu et al., "11 TOPs photonic convolutional accelerator for optical neural networks", Nature, vol. 589, 44-51 (2021).

104. C. Reimer, et al., "Integrated frequency comb source of heralded single photons," Optics Express, vol. 22, no. 6, pp. 6535-6546, 2014.

105. C. Reimer, et al., "Cross-polarized photon-pair generation and bi-chromatically pumped optical parametric oscillation on a chip", Nature Communications, vol. 6, Article 8236, 2015. DOI: 10.1038/ncomms9236

106. L. Caspani, C. Reimer, M. Kues, et al., "Multifrequency sources of quantum correlated photon pairs on-chip: a path toward integrated Quantum Frequency Combs," Nanophotonics, vol. 5, no. 2, pp. 351362, 2016.

107. C. Reimer et al., "Generation of multiphoton entangled quantum states by means of integrated frequency combs," Science, vol. 351, no. 6278, pp. 1176-1180, 2016.

108. M. Kues, et al., "On-chip generation of high-dimensional entangled quantum states and their coherent control", Nature, vol. 546, no. 7660, pp. 622-626, 2017.

109. P. Roztocki, M. Kues, C. Reimer, B. Wetzel, S. Sciara, Y. Zhang, A. Cino, B. E. Little, S. T. Chu, D. J. Moss, and R. Morandotti, "Practical system for the generation of pulsed quantum frequency combs," Optics Express, vol. 25, no. 16, pp. 18940-18949, 2017.

110. Y. Zhang, et al., "Induced photon correlations through superposition of two four-wave mixing processes in integrated cavities", Laser and Photonics Reviews, vol. 14, no. 7, pp. 2000128, 2020. DOI: 10.1002/lpor.202000128

111. M. Kues, C. Reimer, A. Weiner, J. Lukens, W. Munro, D. J. Moss, and R. Morandotti, “Quantum Optical Micro-combs", Nature Photonics, vol. 13, no.3, pp. 170-179, 2019.

112. C. Reimer, et al.,"High-dimensional one-way quantum processing implemented on d-level cluster states", Nature Physics, vol. 15, no.2, pp. 148-153, 2019.

113. T.Ido, H.Sano, D.J.Moss, S.Tanaka, and A.Takai, "Strained InGaAs/InAIAs MQW electroabsorption modulators with large bandwidth and low driving voltage", IEEE Photonics Technology Letters, Vol. 6, 1207 (1994). DOI: 10.1109/68.329640.

114. D. J. Moss, M. Lamont, S. Mclaughlin, G. Randall, P. Colbourne, S. Kiran and C. A. Hulse,"Tunable dispersion and dispersion slope compensators for $10 \mathrm{~Gb} / \mathrm{s}$ using all-pass multicavity etalons", IEEE Photonics Technology Letters, vol. 15, pp. 730, 2003.

115. L. M. Lunardi, D. Moss, S. Chandrasekhar, L. L. Buhl, A. Hulse, P. Colbourne, G. Randall, S. Mclaughlin, "Tunable dispersion compensators based on multi-cavity all-pass etalons for $40 \mathrm{~Gb} / \mathrm{s}$ systems", IEEE J. Lightwave Technology, vol. 20, pp. 2136, 2002. 
116. D. J. Moss, et al., "Multichannel tunable dispersion compensation using all-pass multicavity etalons", TuT2 Optical Fiber Communications Conf., Anaheim (2002). Technical Digest (IEEE Cat. No.02CH37339). Opt Soc. America. Part vol.1, 2002, pp. 132-3. Washington, DC, USA.

117. D. J. Moss, et al., "Tunable dispersion compensation at $10 \mathrm{~Gb} / \mathrm{s}$ and $40 \mathrm{~Gb} / \mathrm{s}$ using multicavity allpass etalons", Optical Fiber Communications Conf. TuD1, p162, Atlanta, GA, March (2003). Digest (IEEE Cat. No.03CH37403). OSA. Part vol.1, 2003, pp. 162-3. Washington, DC, USA.

\section{Figures}

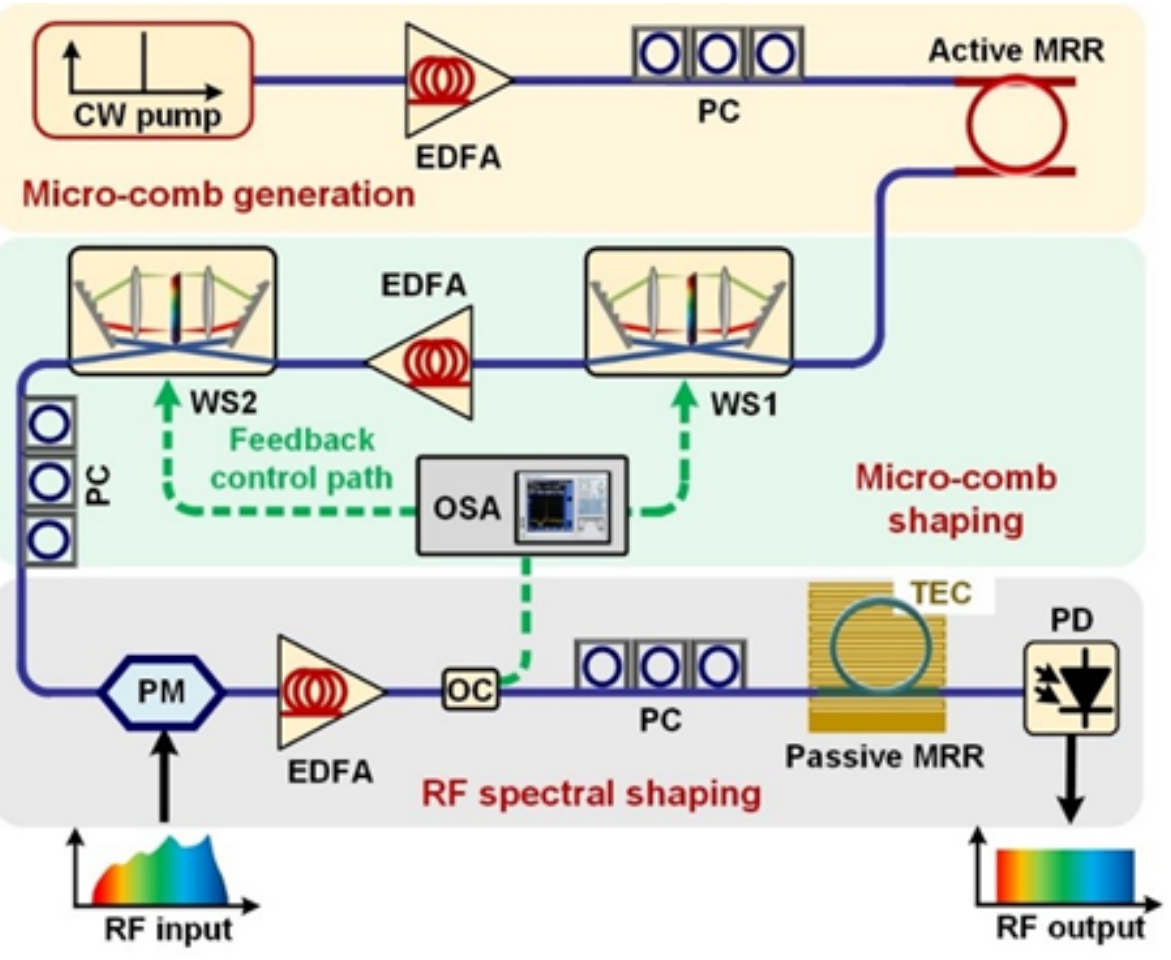

Figure 1

Schematic diagram of the microcomb-based RF filter. EDFA: erbium-doped fiber amplifier. PC: polarization controller. MRR: micro-ring resonator. WS: Waveshaper. PM: Phase modulator. OC: optical coupler. OSA: optical spectrum analyzer. TEC: Thermoelectric cooler. PD: photodetector.
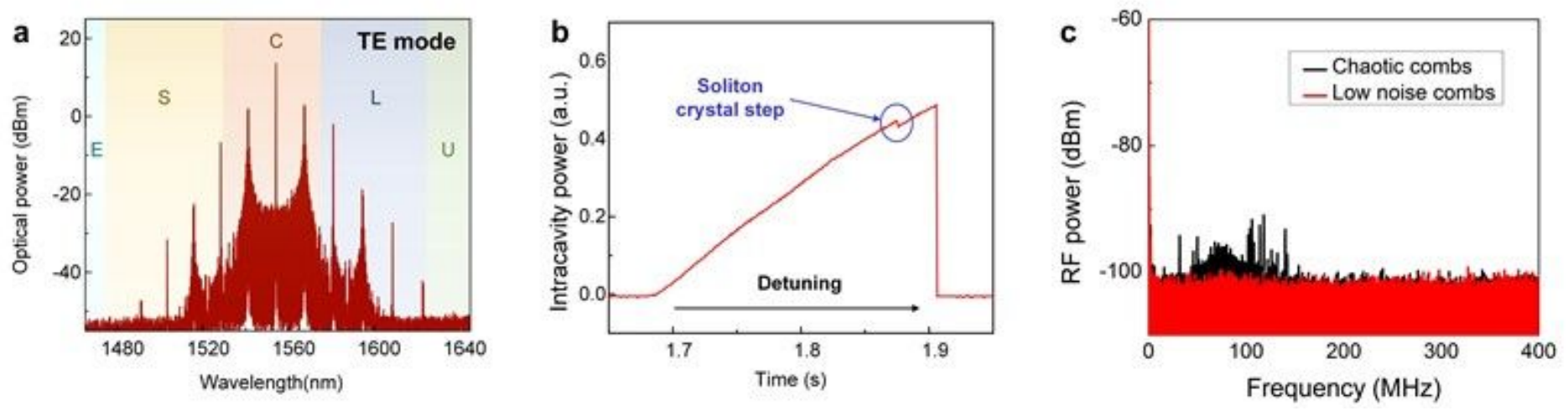
Figure 2

(a) Optical spectrum of the generated micro-combs with a $200 \mathrm{~nm}$ span. (b) Measured transmission of a single resonance showing the soliton crystal step, and (c) the measured RF spectra.
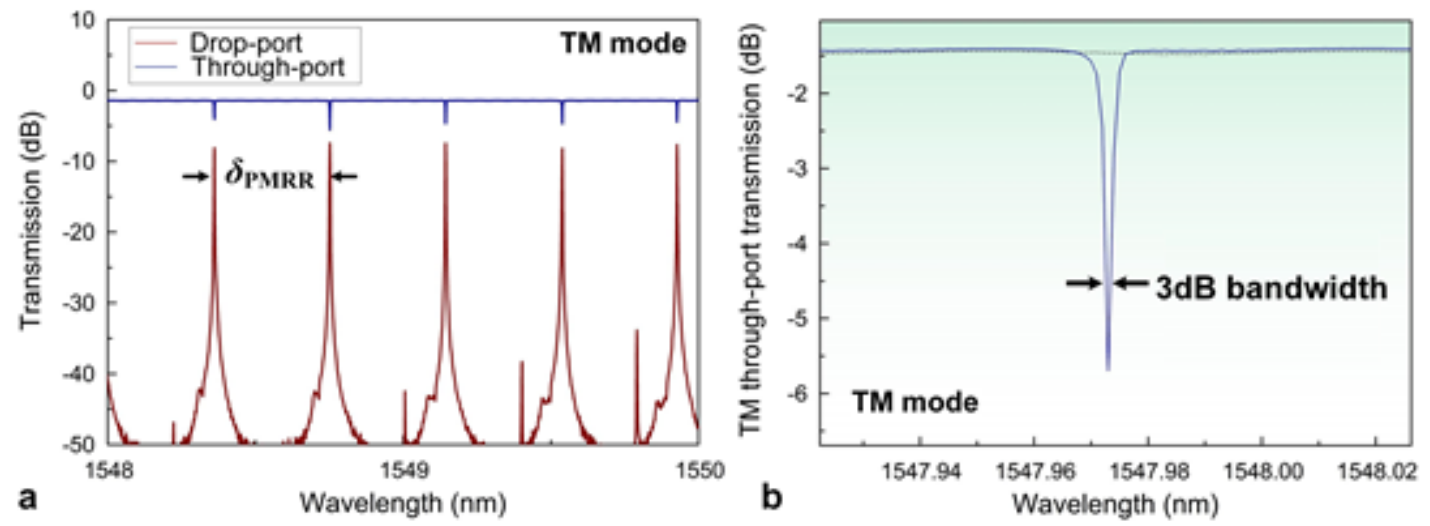

Figure 3

The TM mode through-port transmission spectra of the passive MRR (a) with a span of $5 \mathrm{~nm}$, (b) showing a resonance at 1547.972 with a $3 \mathrm{~dB}$ bandwidth of $\sim 0.84 \mathrm{pm}$, or $\sim 105 \mathrm{MHz}$. The drop-port was not used but just shown here for completion.
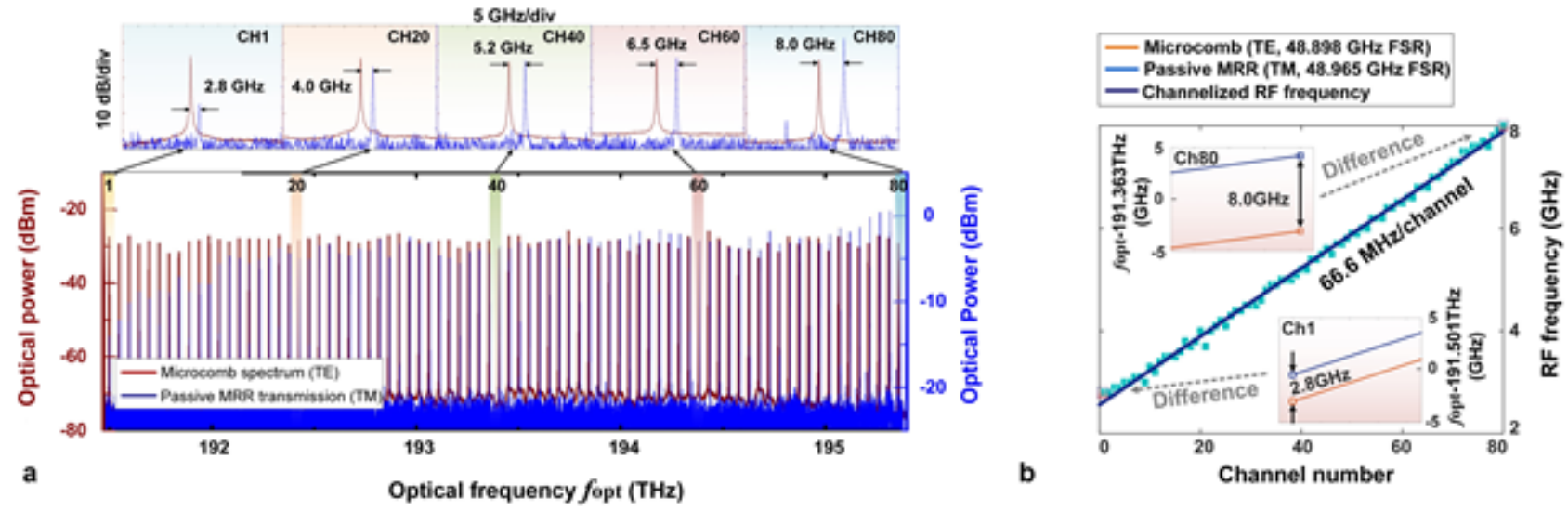

\section{Figure 4}

(a) Measured optical spectrum of the generated micro-comb and transmission spectra of the passive MRR. Zoom-in views of the channels with different channelized RF frequencies. (b) Extracted RF frequencies of the channelized RF spectral segments. 


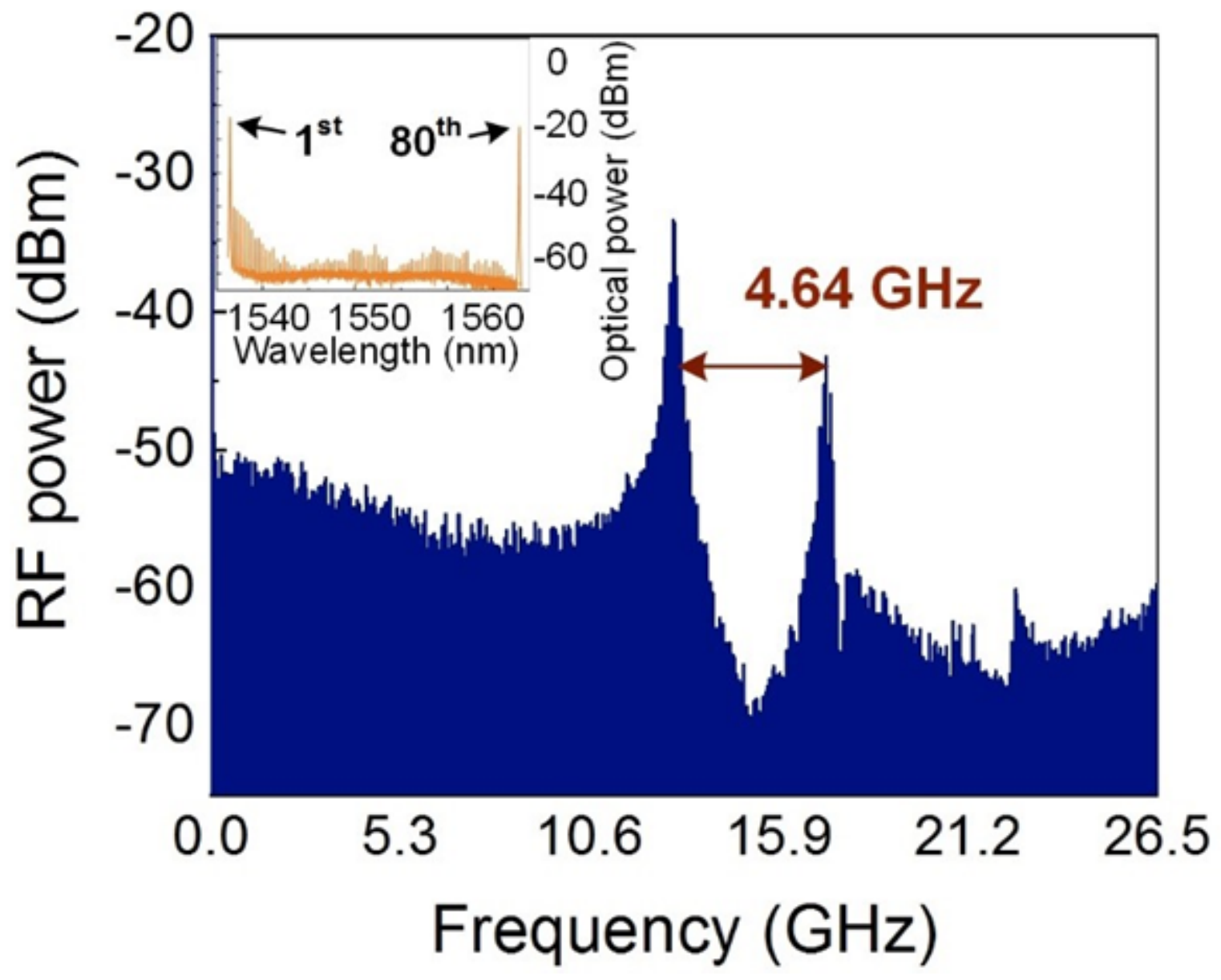

Figure 5

Measured RF transmission spectra of the RF filter with the 1st and 80th comb line being employed. The inset shows the corresponding optical spectrum.

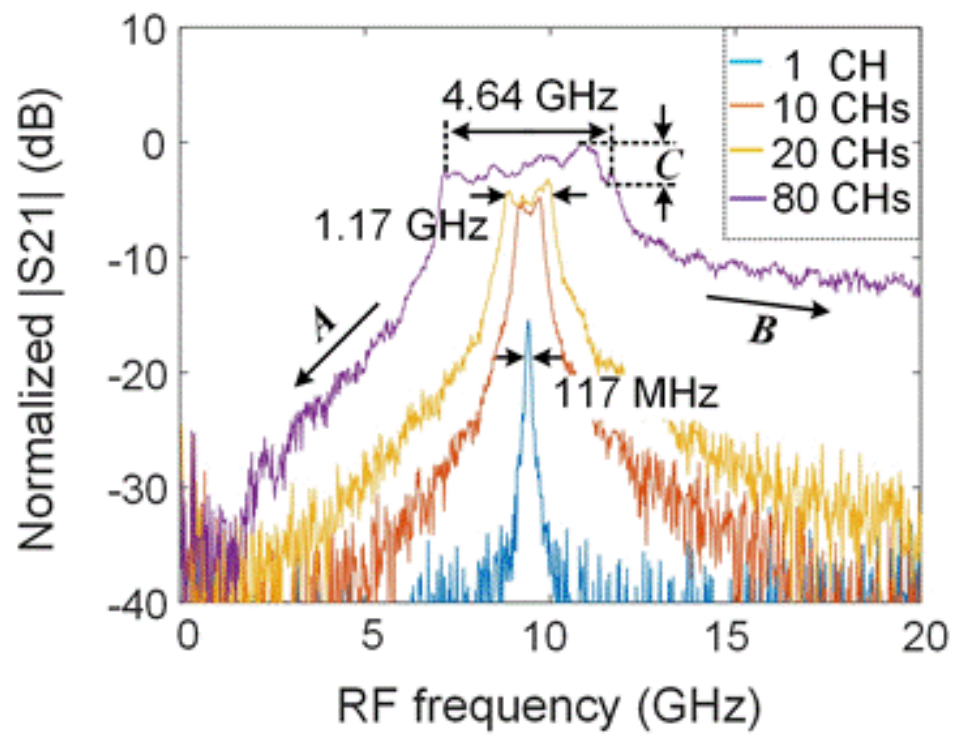

Figure 6 
Measured RF transmission spectra of the RF filter as the number of wavelength channels changed from 1 to 80 . A: roll-off rate $=5.2 \mathrm{~dB} / \mathrm{GHz}$. B: roll-off rate $=1.3 \mathrm{~dB} / \mathrm{GHz}$. C: roll-off rate $=2.9 \mathrm{~dB} / \mathrm{GHz}$.
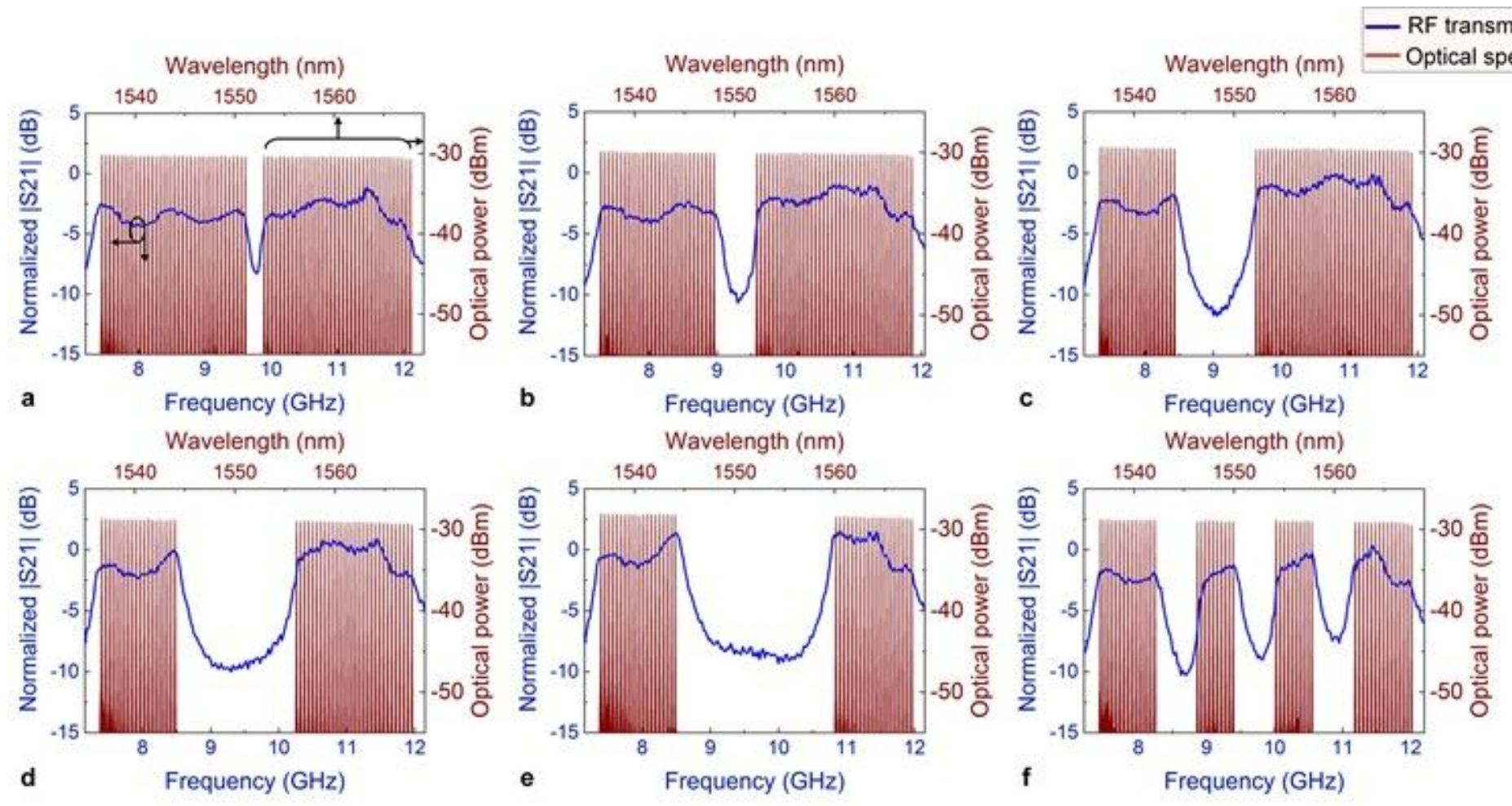

Figure 7

Measured RF transmission spectra (blue line) of the RF filter featuring binary coded channel weights and corresponding optical spectra (red line) of the shaped micro-comb. 

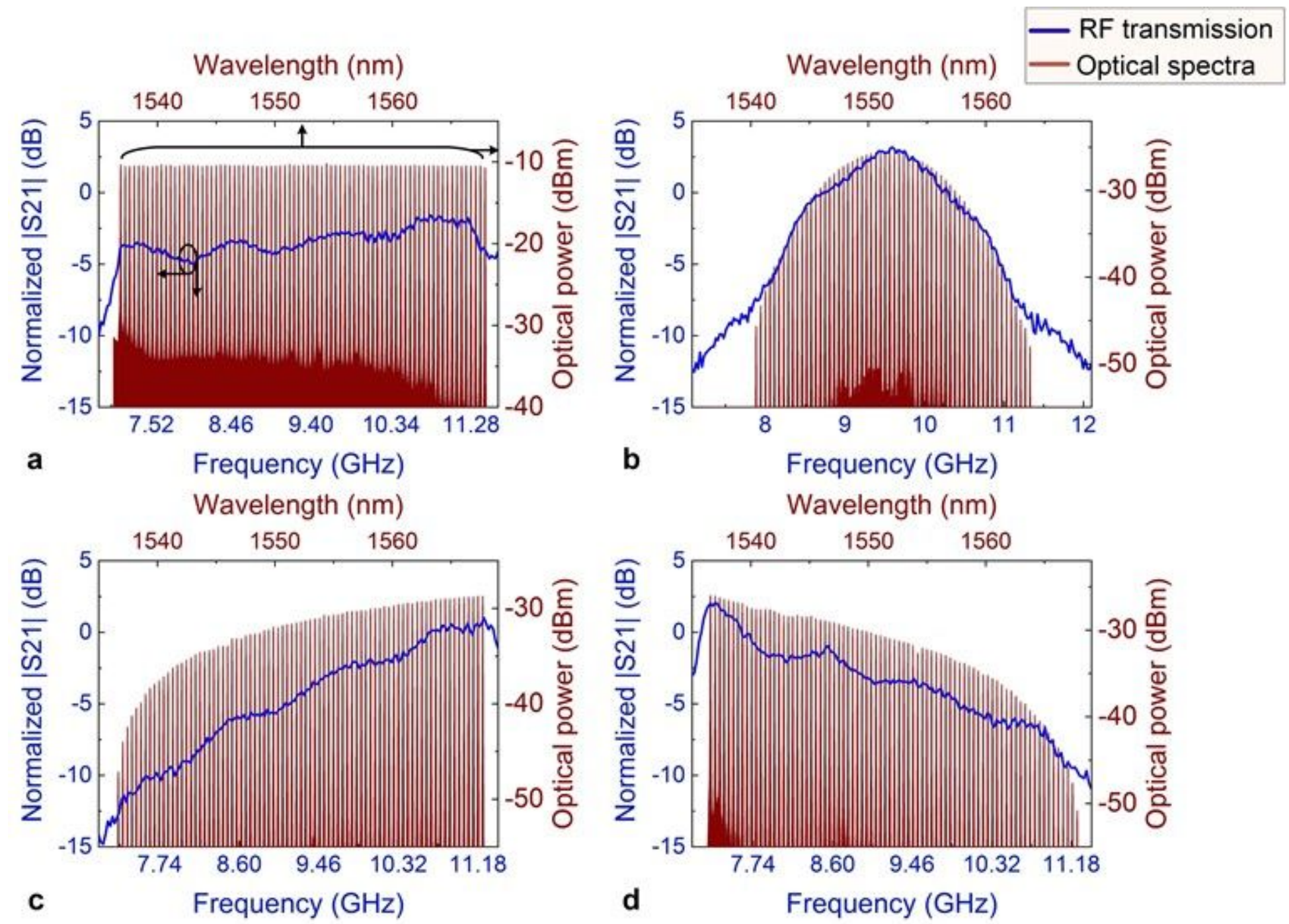

Figure 8

Measured RF transmission spectra (blue line) of the RF equalizing filter featuring varying slopes and corresponding optical spectra (red line) of the shaped micro-comb. 


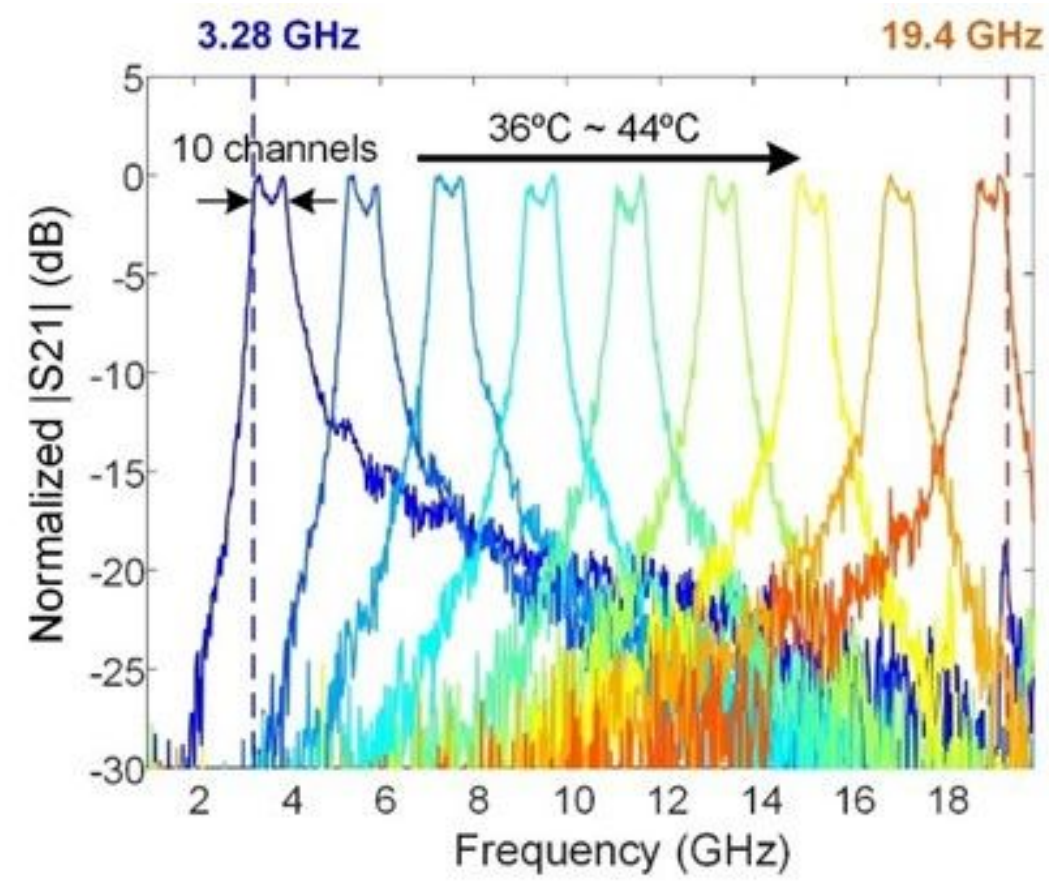

Figure 9

Measured RF transmission spectra of the RF filter with a varying chip temperature of the passive MRR. 